\section{Kramer-Pollnow-Preis 2013}

Der Kramer-Pollnow-Preis (KPP) für besondere wissenschaftliche Leistungen in der klinischen Forschung zur biologischen Kinder- und Jugendpsychiatrie, insbesondere zur Aufmerksamkeitsdefizit-/Hyperaktivitätsstörung wird 2013 zum zehnten Mal und erstmals europaweit ausgeschrieben. Stifter des mit 6.000€ dotierten Preises ist das Unternehmen Medice. Die Ausschreibung läuft bis zum 30. September 2013. Weitere Informationen und Bewerbungsunterlagen bei Professor Rothenberger, E-Mail: arothen@gwdg.de

Nach Informationen von Medice

\section{Tagesschläfer aufwecken}

Exzessive Tageschläfrigkeit ist bei Narkolepsie-Patienten ein häufiges Symptom. Bisher existieren nur symptomatische Therapieansätze. Mit Modafinil (z. B. Vigil ${ }^{\oplus}$ ), das zur Behandlung der Narkolepsie mit und ohne Kataplexie bei Erwachsenen zugelassen ist, steht eine sinnvolle Behandlungsoption zur Verfügung. Laut Leitlinien der Deutschen Gesellschaft für Schlafforschung und Schlafmedizin DGSM ist Modafinil zur Behandlung der Tagesschläfrigkeit bei Narkolepsie das Mittel der ersten Wahl.

Nach Informationen von Teva

\section{Mehr als Medikamente}

Die 2013 gegründete neurax-Foundation hat sich zum Ziel gesetzt, Fachkräfte, Patienten und Angehörige zu unterstützen. Ein Schwerpunkt des Engagements ist die pädiatrische Neurologie und Psychiatrie mit der tiergestützten Therapie. Mit über 1 Million $€$ bezuschusst neuraxpharm aktuell die Errichtung des Zentrums für tiergestützte Therapie in Stadtbergen bei Augsburg. Kinder und Jugendliche mit psychologischen und somatisch-neurologischen Störungen sowie traumatisierte Kinder können dort ab 2014 ambulante sowie stationäre Unterstützung mit ihren Familien in Anspruch nehmen.

Nach Informationen von neuraxpharm

Schizophrenie

\title{
Häufiger und früher langwirksame Antipsychotika einsetzen!
}

Ein Hauptproblem der Therapie der Schizophrenie ist die mangelhafte Therapieadhärenz. Lang wirksame (Depot-) Antipsychotika haben hier entscheidende Vorteile und können so auf längere Sicht die notwendige Rezidivprophylaxe gewährleisten.

„Die Behandlung der Schizophrenie entscheidet sich erst auf lange Sicht“, betonte Professor Hans-Peter Volz, Ärztlicher Direktor des Krankenhauses für Psychiatrie, Psychotherapie und Psychosomatische Medizin Schloss Werneck. Dabei sieht der Experte einen Vorteil für lang wirksame Antipsychotika wie Paliperidonpalmitat (Xeplion ${ }^{\circledast}$ ). Dafür sprechen auch die Ergebnisse populationsbasierter Studien, wie Professor Stefan Leucht, stellvertretender Direktor der Klinik für Psychiatrie und Psychotherapie der TU München, erläuterte. Bei Anwendung von Depot-Antipsychotika reduzierte sich in einer finnischen Registerstudie beispielsweise die Rehospitalisierungsrate gegenüber oralen Substanzen signifikant um zwei Drittel [Tiihonen J et al. BMJ 2006; 333: 224; Tiihonen J et al. Am J Psychiatry 2011; 168: 603 -9].

\section{Erhaltungsphase für Optimierung nutzen}

Für die Langzeittherapie empfehlen die Leitlinien [Gaebel et al. S3-Behandlungsleitlinie Schizophrenie. Steinkopff Verlag Darmstadt 2006] eine Monotherapie mit dem Antipsychotikum, das sich auch in der Akuttherapie als wirksam und verträglich erwiesen hat. Allerdings werden in der Akuttherapie häufig Kombinationen eingesetzt und die empfohlenen Dosierungen weit überschritten. Die Erhaltungstherapie sollte deshalb dazu genutzt werden, die Behandlung auf eine Monotherapie in adäquater Dosis zu reduzieren, riet Professor Göran Hajak, Direktor der Klinik für Psychiatrie, Psychosomatik und Psychotherapie der Sozialstiftung Bamberg. Dabei sollte immer auch eine Depotmedikation in Betracht gezogen werden. In psychiatrischen Ambulanzen wachse der Einsatz von lang wirksamen Depot-Antipsychotika, manche Einrichtungen setzten fast nur noch solche lang wirksamen Antipsychotika ein, berichtete Hajak. Patienten, die Depot-Antipsychotika wie Paliperidonpalmitat erhielten, seien meist die besser geführten Patienten. Die Compliance sei gut überprüfbar und die monatliche Injektion könne mit einem therapeutischen Gespräch verbunden werden, sodass aus der monatlichen Vorstellung ein echter therapeutischer Vorteil entstünde. Deshalb schützten lang wirksame Antipsychotika besser vor Rückfällen, sagte Hajak. „Meiner Erfahrung nach ist die Versorgung der Patienten mit Depotmedikation einfach besser."

Friederike Klein, freie Medizinjournalistin

3. Bamberger CNS-Summit, 13.4.2013

Veranstalter: Janssen-Cilag

\section{Alle Dimensionen der Erkrankung therapeutisch adressieren}

Patienten mit Depressionen sind nicht nur durch eine gedrückte Stimmung in ihrem Alltagsleben beeinträchtigt, sondern vor allem auch durch den Verlust positiver Emotionen und Defizite in der psychosozialen Funktionsfähigkeit. Im klinischen Alltag werden diese Aspekte bei der Diagnose der Depression als auch bei der Bewertung der Remission nur unzureichend berücksichtigt, meinte Professor Koen Demyttenaere, Universitätsklinik Gasthuisberg in Leuven/Belgien. Denn mit üblichen Depressionsskalen werde lediglich die depressive Symptomatik erfasst, nicht aber Beeinträchtigungen der Alltagsfunktionen. Da die 